\title{
Estadísticas de turistas en laguna de Quilotoa
}

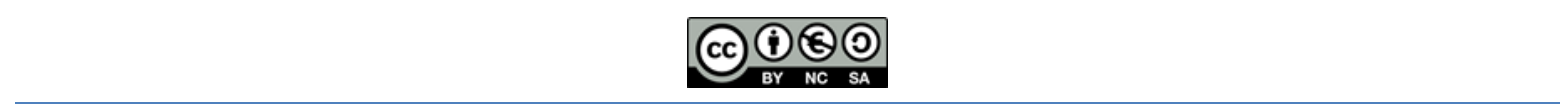

\section{Quilotoa Lagoon Tourist Statistics}

\author{
Yolanda Tatiana Carrasco Ruano. ${ }^{1}$
}

Resumen.

DOI: https://doi.org/10.33262/concienciadigital.v2i1.927

Muchos años más antes, el lugar que hoy se encuentra la Laguna, ha sido una planicie de llanura con muchos pastizales y plantas frutales como: caña, mandarina, naranja, maíz entre otros.

En medio de tantos árboles frutales, madereros y vegetales, ha existido árbol muy grande con sus ramas formadas como dientes y muy hermosas.

He ahí, ha vivido un vaquero cuidando a los ganados, en ese entonces ha existido una vertiente de agua que ha estado ubicado en tuya chupa, que en la actualidad en ese lugar existe agua termal que está burbujeando.

En una semana o más el agua se ha comenzado a salir más y en sueño se ha presentado una chica muy linda brillando junto al árbol grande y se ha revelado diciendo; que hoy saldrá más agua, formaré los picos y cuchillas.

Luego de esta revelación, el vaquero se ha asustado muchísimo por no interpretar que quería decir esa revelación; así ha comenzado a difundir a una familia que ha existido en ese entonces en zona de Quilotoa.

También hay otras muchas versiones, pero de todo, el nombre kirutwa se origina de esa revelación y versión, que hace mucho más año que nuestros abuelas/as y ancestros siempre ha llamado kirutwa, que significa: kiru = dientes o madera y twa = princesa, es por ello el significado de kirutwa es dientes de princesa toa.

\footnotetext{
${ }^{1}$ Ciencia digital, Ambato, Ecuador, tcarrascor@yahoo.com
} 
Ahora en la actualidad con la llegada de mestizos y más personas extranjeras, se ha cambiado de nombre de Kirutwa a Quilotoa que no tiene mayor significado para nosotros.

Palabras claves: Laguna, Quilotoa, Kirutwa.

\section{Abstract.}

The Quilotoa lagoon is one of the 15 lagoons of volcanic origin appreciated as the most beautiful in the world according to Twistedsifter. In the article published by this website with the name " 15 of the most beautiful volcanic lakes in the world" readers are invited to enjoy this mysterious wonder of nature through a photographic gallery that describes the lagoons according to their charm.

The Quilotoa lagoon and the beauty inside a crater Quilotoa is the westernmost volcano of the Ecuadorian Andes, within its crater a caldera has formed with a diameter of almost $9 \mathrm{~km}$. This is the product of the collapse of the volcano, about 800 years ago. According to studies, the volcanic flow will reach the Pacific Ocean.

In the $250 \mathrm{~m}$ deep boiler, a lagoon has formed. The minerals have given the surface a greenish and bluish tone when it receives the impact of sunlight. Inside, there are springs that from the shore, you can perceive as bubbles of light trails that ascend to the surface.

The popularity of the Quilotoa must be the beauty of its places and the spectacle of the calm waters. In the community of Quilotoa, also known as the Route of the "Summit" and which is accessed by bus from the town of Zumbahua, visitors can do ecotourism and adventure tourism with a high dose of adrenaline or simply contemplate the landscapes.

This is the reason why the last route of Inca Atahualpa has been used, as well as the last sacred route of the Incas. Visiting Quilotoa is an overwhelming experience at approximately 4 thousand meters above sea level.

Keywords: Economy, Galapagos, flora, fauna, direct, indirect, types of tourism.

\section{Introducción.}

Según (osorio, 2014)Uno de los atractivos naturales más importantes en Cotopaxi A 66 km de Latacunga está una de las maravillas naturales más impresionantes de Cotopaxi: la laguna del Quilotoa. Allí, el turismo comunitario se convirtió en una forma de vida. Más de 1.000 visitantes arriban mensualmente. 
A 3.800 metros de altitud, en una parte de la Reserva Ecológica Los Illinizas, en la parroquia Zumbahua (Pujilí), se encuentra el complejo turístico del Quilotoa, que incluye un centro de artesanías, hosterías, un parqueadero, senderos, zona de camping y paseos en kayak. En la zona artesanal hay más de 30 puestos en los que se venden bufandas, guantes, gorras, suéteres y chalinas, elaborados con lana de alpaca. También hay llaveros, esteras, cuadros y caretas fabricadas por los artesanos de Tigua. Esta construcción, de una sola planta, guarda el encanto de las casas de teja que abundan en los poblados cercanos. "Los sábados, domingos y días feriados son los de mayor concurrencia. De lunes a viernes el negocio decae", dijo María Toaquiza, cuyas ventas mayores (80\%) se concretan con turistas extranjeros. Para descender del complejo hacia las turquesas aguas de la laguna, que está dentro del cráter del volcán, hay que caminar por un sendero, por cerca de 40 minutos. El espejo del agua tiene 3 kilómetros y una profundidad promedio de 240 metros.

En el entorno, los comuneros se organizaron y con la colaboración del Ministerio de Ambiente pusieron en marcha el proyecto de paseo en kayak (canoa individual de remo). Hay 5 de estas naves y el paseo de media hora cuesta \$2,50. La turista quiteña Andrea Silva probó el viaje y dijo que era maravilloso. "Los colores de la laguna varían de acuerdo con la intensidad del Sol", manifestó. Para ascender el largo y empinado sendero, los comuneros cuentan con mulas y caballos que alquilan a $\$ 10$. "Cada animal es capaz de realizar hasta 20 carreras diarias", aseguró Miguel T.

Pero no todos están de acuerdo con este medio de transporte. Algunos opinan que es inhumano forzar a las mulas a emprender este duro y empinado camino. Otros prefieren efectuar el ejercicio. "Creo que sería una maravillosa idea que se colocara una tarabita. Así los comuneros podrían atraer más turismo y se protegería a los animales de tanto desgaste", sugirió Graciela Proaño, turista.

\section{Materiales y métodos}

\section{Estadísticas en el año 2015, 2016, 2017 y 2018}

Según (travel, 2015): USD 1.691,2 millones (e) en el año 2015 por ingreso de divisas.

$\mathrm{Al}$ año 2015 el país registra por cuarto año consecutivo superávit en la balanza turística.

Al año 2015 se registró un saldo positivo en la balanza turística estimado en USD 650 millones.

\section{Laguna Quilotoa considerada una de las lagunas más bellas del Mundo}

Según (Twistedsifter, 2017). La Laguna del Quilotoa es una de las 15 lagunas de origen volcánico apreciadas como las más hermosas del mundo. En el artículo publicado por este sitio web con el nombre " 15 de los más hermosos lagos volcánicos en el mundo" se invita a 
los lectores a disfrutar de esta misteriosa maravilla de la naturaleza mediante una galería fotográfica que describe a las lagunas según su encanto.

La Laguna del Quilotoa y la belleza dentro de un cráter.

El Quilotoa es el volcán más occidental de los Andes ecuatorianos, dentro de su cráter se ha formado una caldera con un diámetro de casi $9 \mathrm{~km}$. Este es el producto del colapso del volcán, ocurrido hace unos 800 años. Según estudios, el flujo volcánico logró alcanzar el Océano Pacífico.

En la caldera de $250 \mathrm{~m}$ de profundidad, se ha formado una laguna. Los minerales le han conferido a la superficie un tono verdoso y azulado cuando recibe el impacto de la luz solar. Dentro, existen manantiales que desde la orilla, se pueden percibir como burbujeantes estelas que ascienden a la superficie.

La popularidad del Quilotoa se debe a la belleza de sus parajes y el espectáculo de las aguas calmas. En la comunidad de Quilotoa, también conocida como la Ruta de la "Cumbre" y a la que se accede en bus desde el pueblo de Zumbahua, los visitantes pueden realizar ecoturismo y turismo de aventura con una alta dosis de adrenalina o simplemente contemplar los paisajes cercanos.

Incluso este paraje es cercano a lo que muchos investigadores asumen como la última morada del Inca Atahualpa, por lo que esta fue la última ruta sagrada de los incas. Visitar Quilotoa es una experiencia sobrecogedora aproximadamente a 4 mil metros sobre el nivel del mar.

\section{Datos Informativos}

\section{¿Cómo llegar?}

Los turistas que visitan el volcán y la laguna del Quilotoa utilizan las frecuencias de buses que diariamente y a diferentes horas salen hacia Latacunga y desde esta ciudad, utilizan los servicios que enlazan Latacunga, Pujilí, Zumbahua $(64,33 \mathrm{~km})$ y desde esta población al Quilotoa (12,67 km). El tiempo aproximado de llegada desde Latacunga hasta el Mirador de Quilota es de 1 hora.

\section{¿Dónde hospedarse?}

En las comunidades Quilotoa, Zumbahua, Tigua, Shalalá, Chugchilán, Itupungo y Guayama San Pedro se ofrece alojamiento de distintos tipos, como hosterías, hoteles, cabañas, hostales y posadas. También alquilan caballos y medios de transporte acuático como kayaks y botes, respaldando la práctica de las actividades deportivas que se realizan en los radios de influencia del Circuito Turístico Quilotoa, como Trecking, Hiking, Cabalgata, Camping y Rodeo de altura. 
Actividades

Trecking, hiking, cabalgata, camping y rodeo de altura.

Temperatura

Entre 3 y 15 grados centígrados

Sitios de interés turístico

Latacunga / Pujilí / Tigua / Quilotoa / Sigchos / Saquisilí.

\section{Mi criterio}

Cuando los primeros fundadores y más personas que iniciaron a desarrollar la feria de artesanas, se surgió la necesidad de conformar una organización o asociación con personería jurídica; Es por ello en año 1991 se creó una asociación denominado “Asociación de Pintores y tejidos de Quilotoa", conformado con 27 socios.

Luego, por ingreso de varias personas a la asociación y aumento demográfico de las personas, surgió otra necesidad de ampliar el nombre, fines, objetivos y metas de la asociación. Es por ello, en 2007 se conseguido su personería jurídica denominando Organización de Turismo Comunitaria de Desarrollo Turístico Lago Verde Quilotoa, reconocido por el Ministerio de Turismo del Ecuador. Al momento esta organización agrupa 120 socios.

Primera opción. - De la ciudad de Latacunga tomar la vía Pujilí - Zumbahua - Quevedo. Cuando viajas en los transportes como: Cotopaxi, La Mana, el Ciro, Ambato, Pujilí, Salcedo, Cevallos y 14 de Octubre. La primera es en Campamento -Zumbahua, luego puede transitar en camionetas hasta Quilotoa.

Segunda opción. - Desde el Terminal de la ciudad de Latacunga puede tomar el viaje directo hasta Quilotoa las siguientes cooperativas de transportes: Vivero que llega al cráter de la Laguna y transporte Iliniza que llega hasta parroquia Chugchilan.

\section{Organización Socio-Política}

Está compuesta de pequeñas comunidades indígenas que viven de la agricultura a una altura de 4.000 m.s.n.m. Son descendientes de las comunidades Panzaleos que vivían en esas zonas. Quilotoa fue fundada hace solo ocho años por los residentes del pueblo de Ponce, situado a de 2002.

La Organización no posee un sistema de políticas administrativas claras ni bien definidas, en parte por la carencia de educación. Dentro de este lugar prima la desorganización y la desunión entre sus miembros, resulta difícil concretar planes, proyectos. 


\section{Economía}

Cerca de Zumbahua usted encontrará Tigua, un pequeño pueblito con una comunidad dedicada a producir pinturas "naïf" no en su base tradicional sino en piel seca de oveja. Estas pinturas son muy coloridas, y representan la tierra y costumbres de la gente que vive en la región de Quilotoa.

La población se dedica a la agricultura con fines comerciales y de subsistencia.

El más cercano centro para la comercialización agraria se encuentra en Zumbahua, y la comunidad sigue utilizando esta mercado para vender sus productos. Sin embargo, la mayoría de los entrevistados explicó que la producción ha disminuido debido a malas condiciones del suelo y la ausencia de condiciones de mercado favorables.

\section{Educación}

En septiembre del 2008 se abrió la Unidad Educativa Cacique Tumbalá siendo la primera de las diez Unidades Educativas del Milenio que se construyen a nivel nacional y que cuenta con características singulares en cuanto a la oferta y al modelo educativo inclusivo, para el servicio de 925 niños, niñas y jóvenes de este sector en los niveles inicial, general básica y bachillerato en tres idiomas: quichua, castellano e inglés.

\section{Vivienda}

La gente vive en viviendas tradicionales, en sus chozas. Aunque en los últimos años ha habido un cambio acelerado por la sustitución de casas de bloque, cemento y zinc, muy desfavorables por las condiciones climáticas del lugar.

\section{Vestimenta}

Antiguamente el hombre usaba pantalón blanco y camisa del mismo color, pero de liencillo; que ellos mismos confeccionaban, también ponchos rojos, bufanda a su gusto de lana de borrego y sombrero blanco de la misma lana.

La mujer usa hasta ahora saco de lana de borrego negro, bayeta de color con tupu (agujon), arretes con monedas antiguas, el cabello amarrado con lana de llamingo haciendo chimba (trenza). Tanto hombre como la mujer no utilizaban zapatos y andaban a pie descalzo. Hoy todo está modernizado: hombres con chompas, zapatos de cuero, pantalones confeccionados por otros que saben de su profesión.

\section{Actividades Turísticas}

Turismo en la Laguna: De las conversaciones con los propietarios de los operadores turísticos en la región y el pionero de indígenas familias involucradas en el negocio, es posible concluir que la región comenzó a atraer a los visitantes. 
Cada comunidad se especializa en diferentes lugares de interés cultural y natural, tales como indígenas, festivales, mercados indígenas, el trabajo artesanal, comidas tradicionales y tradicionales indígenas en general, vida. Entre los atractivos naturales encontramos irregulares paisajes de montaña, los mosaicos de la agricultura parches, fuentes termales, ruinas arqueológicas, los indígenas y la arquitectura vernácula, entre el más importante.

La mayoría de la actividad turística no es controlada por el gobierno, sin embargo, bajo ciertas circunstancias, locales de en ocasiones los gobiernos intervenir. La presencia indígena es elevada en la región y locales cabildos supervisar la entrada de visitantes a la zona. Cabildos asegurar que la actividad turística se no presenta ninguna amenaza para su supervivencia, pero se convierte en una oportunidad económica. Comunas beneficio del turismo en función del grado de participación en la prestación de servicios.

Alojamiento y proveedores de alimentos se concentran en la ciudad de Latacunga.Albergues dentro de la región son pequeños en número. Durante el trabajo de campo fue posible identificar ocho hostales en el corredor turístico antes mencionado.

En gran parte, los mestizos o los empresarios extranjeros que han penetrado en el área propia de estos negocios.

En este sentido, es importante señalar que la comuna en Quilotoa ha logrado el desarrollo de la todos los servicios turísticos a nivel local. En otras palabras, diferentes de todas las comunas, las prestaciones del Quilotoa de la plena participación en la prestación de todos los servicios con las limitaciones en el ámbito del transporte.

\section{Tours:}

Según (time, 2017)Organización Comunitaria de Desarrollo Turístico Lago Verde Quilotoa, por medio de sus guías nativos ofrece a realizar los siguientes tours a lugares atractivos y sagrados.

De Quilotoa alrededor del cráter, caminata 5 a 6 horas de todo el radio.

Del cráter al descenso a la Laguna 30 minuto y ascenso 45 minutos o una hora de caminata. Servicio de acémilas para el ascenso de la Laguna al cráter y a otros lugares.

Desde Quilotoa a Cueva del Inca o Mama Jatava con acémilas de ida y vuelta caminata 4 horas y con acémila 1 hora 20 minutos

Desde Quilotoa al río Toachi - Zumbahua en caminata 4 horas.

Desde Quilotoa a aguas termales en caminata de ida y vuelta 6 horas y en acémilas 5 horas.

De Quilotoa a Chugchilan en caminata 4 a 5 horas y en acémala 2 horas.

Para realizar una caminata con acémilas desde Quilotoa hasta cantón Pujilí con carga, tiene los siguientes recorridos:

Primer día.- Desde Quilotoa se llega hasta Rumi Pamba (alojamiento) 
Segundo día.- De Rumi Pamba a Akchi Vaquería (alojamiento)

Tercer día.- De Akchi Vaquería a cantón Pujilí

Para realizar una caminata desde Quilotoa hasta las Pampas que esta ubicado en cantón Sigchos con carga, tiene los siguientes recorridos:

Primer día.- desde Quilotoa se llega hasta el Itualo

Segundo día.- De Itualo a Siwicosi

Tercer día.- Siwicosi a la Chorrera

Cuarto día.- De Chorrera a Sachis

Quinto día.- De Sachis a San Pablo

Sexto día.- De San Pablo a las Pampas

NOTA: los puntos de alojamiento o dormida es en cada sitio antes en mención.

El Tours que se realiza desde Quilotoa a Angamarca y punto de recogida en Zumbahua, tiene los siguientes recorridos:

Primer día.- desde Quilotoa se llega hasta comuna Apahua

Segundo día.- De Apahua a Chixta loma

Tercer día.- De Chixta loma a parroquia Angarmarca (alojamiento)

Cuarto día.- De Angamarca a Pigua- Wagracurral (alojamiento)

Quinto día.- De Pigua- Wagracurral a Cóndor Cucha ( alojamiento )

Sexto día.- De Cóndor Cucha a Michacala - Guantopolo y Zumbahua.

El Tours que se realiza desde Quilotoa a Puca Yaco que esta por costa, tiene los siguientes recorridos:

Primer día.- Desde Quilotoa se llega hasta el Niño Urdu (alojamiento)

Segundo día.- De Niño Urdu a Yacu Chaki (alojamiento)

Tercer día.- De Yacu Chaki a parroquia Puca.

\section{Mi criterio:}

Para dichos Tours que ofrece la organización están encargadas las siguientes personas para la coordinación y guiansa:

Juan Cesar Umajinga Umajinga (para comunicar al Cel. 094556944)

Juan Jorge Latacunga Chaluisa (para comunicar al Cel. 085689984)

Juan Alfonso Latacunga Pastuña (para comunicar al Cel. 086059201)

La Organización Comunitaria de Desarrollo Turístico Lago Verde Quilotoa, por medio de sus socios afiliados y seleccionados, tanto hombres y mujeres, están preparados para cumplir la función de guías a los sitios antes en mención, brindando su responsabilidad, seguridad, confianza, brindando la información necesaria - veracidad y también su amabilidad como lo 
caracteriza los indígenas Kichwas.

Figura N 1: Estadísticas desde año 2014, 2015, 2016, 2017, 2018

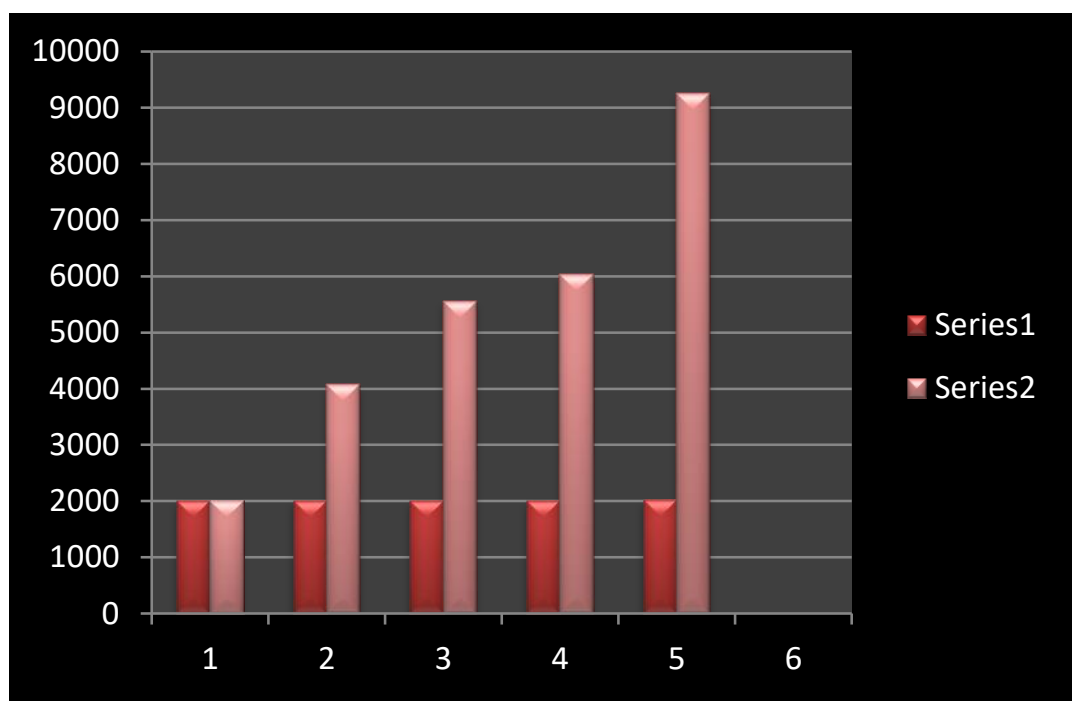

\section{La organización ofrece las siguientes artesanías.}

Según (pilatasig, 2018)Tiene 45 años, ha dedicado a elaborar cuadros, abrigos, guantes y un sinnúmero de recuerdos ofertados a los turistas que acuden cada día hasta la laguna bautizada con el mismo nombre de la comunidad.

Se exhiben máscaras de madera, cuadros pintados con motivos andinos, bateas, gorros tejidos, ropa confeccionada a base de lana de alpaca, chales, cobijas y decenas de objetos.

Cesar Pilataxi es otro comunero que trabaja desde su casa en la elaboración de máscaras que representan a los animales típicos de las festividades andinas, como el cóndor y el lobo, así como también a los bailarines y diablos que son tradicionales en las festividades de Navidad, Corpus Christi o carnaval. Los cuadros en miniatura son pintados con óleo utilizando varias técnicas que gustan al turista y reflejan lo imponente del Cotopaxi, los Illinizas, las lagunas o la cotidianidad del campesino al labrar la tierra y cosechar la cebada.

Recuerda que hace seis años los agremiados con apoyo de la Cooperativa Maquita Cushunchic elaboraron el proyecto para que el lugar sea digno de comercializar de sus artesanías. Antes lo hacían a la intemperie, soportando los intensos fríos y respirando polvo 
provocado por los remolinos de viento que son comunes a los 3.800 metros sobre el nivel del mar.

La cooperativa les implementó telares para que confeccionaran las prendas y al mismo tiempo brindaron capacitación y asistencia técnica a los indígenas de la zona.

Los puestos se ubican en una gran casona construida en la parte alta de la laguna, donde los turistas encuentran las variedades de artesanías elaboradas por los indígenas de la comunidad.

\section{Conclusiones}

- Que todo impacto turístico es muy importante para el ingreso económico en la familia.

- Por medio de narración de historia de la Laguna de Quilotoa promocionamos la hermosura de la naturaleza que existe en esta zona.

- Este trabajo ha sido un esfuerzo y sacrificio, pero con la ayuda de los comuneros y del directivo de la comuna culminado con éxito.

\section{Referencias bibliográficas.}

Osorio, s. (29 de 07 de 2014). https://www.eltelegrafo.com.ec/noticias/regional/1/mas-de-1000-turistas-por-mes-arriban-al-quilotoa.

Pilatasig, m. h. $(10$ de 09 de 2018). Obtenido de http://www.ecotec.edu.ec/documentacion\%5Cactividades_y_eventos/32088_2011_ CEE_DBAQUERIZO_710.pdf.

Time, t. (15 de 05 de 2017). Obtenido de https://traveltime.com.ec/destinos/andes/lagunaquilotoa/tour-a-la-laguna-quilotoa/.

Travel, e. (21 de 07 de 2015). Obtenido de https://www.turismo.gob.ec/wpcontent/uploads/2016/03/RENDICI\%C3\%93N-DE-CUENTAS_MINTUR-1.pdf.

Twistedsifter. (03 de 07 de 2017). https://www.guiadepastaza.com/blogs/detalle/LagunaQuilotoa-considerada-una-de-las-lagunas-mas-bellas-del-Mundo-turismo-ecuador.

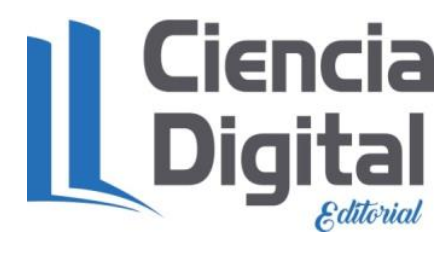


Para citar el artículo indexado.

Carrasco Ruano, Y. (2019). Estadísticas de turistas en laguna de Quilotoa. ConcienciaDigital, 2(1), 24-34. https://doi.org/10.33262/concienciadigital.v2i1.927

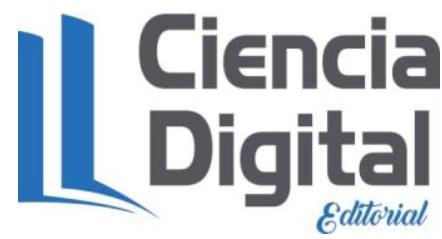

El artículo que se publica es de exclusiva responsabilidad de los autores y no necesariamente reflejan el pensamiento de la Revista Conciencia Digital.

El articulo queda en propiedad de la revista y, por tanto, su publicación parcial y/o total en otro medio tiene que ser autorizado por el director de la Revista Conciencia Digital.
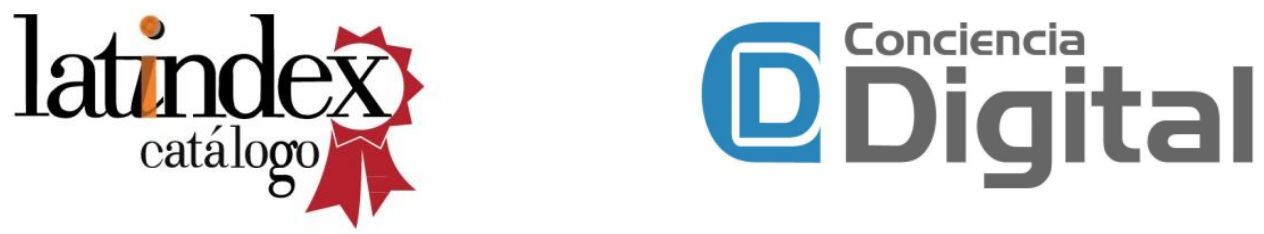